\title{
THE USE OF ASTEROIDS FOR DETERMINATIONS OF MASSES AND OTHER FUNDAMENTAL CONSTANTS
}

\author{
EUGENE RABE \\ Cincinnati Observatory
}

This report does not attempt to review all past and present work using asteroids for determinations of planetary masses and other fundamental constants. This would be a very large undertaking, and too extensively outside of the scope of a colloquium devoted to physical studies of minor planets. It seems preferable to concentrate more on principles involved, while mentioning only some of the various results obtained. At the same time, it will be appropriate to take note of the changed situation resulting from the recent development of certain modern methods and facilities that make it possible to determine some of the constants more accurately in other ways, thus partly eliminating the once dominant role of the minor planets.

\section{DETERMINATION OF PLANETARY MASSES}

Because the orbital motion of any planet or other body in the central gravitational field of the Sun is influenced also by the perturbing gravitational forces of the major planets, it is evident at once that in principle it is possible to determine the masses of these disturbing planets from a careful comparison of the computed and observed motion of the selected object. In most cases, provisional but already fairly good values of the masses, orbital elements, and other unknowns involved can be used as the basis of such a comparison, and thus the quantities to be determined will be corrections to the provisional values. Generally, therefore, it will be sufficient to establish linear observation equations relating the sought-for corrections with the residuals, observation minus calculation, or $O-C$. None of the actual equations relating the various unknowns and the computed positions will be given or derived in this descriptive report, which emphasizes general principles and aspects rather than the too numerous procedural details involved.

The accuracy or even feasibility of any mass determination will depend, of course, aside from the computational or analytical precision of the orbits involved, first, on the magnitude of the perturbations produced by the mass that one wants to improve and, second, on the accuracy as well as the adequate number and distribution of the observed positions. The orbits of the major 
planets themselves, and extended series of observations of the Sun and the principal planets, have been available for a long time and have indeed been used for the determination of improved masses on the basis of their mutual attractions. The attainable accuracy has been rather limited, however, because until recently the degree of precision of the orbital theories of some of the major planets was not very high, and the comparative smallness of the perturbations involved magnified the relative seriousness of orbital theory defects in the resulting mass corrections. Also, on the observational side, the attainable accuracy of positions of the Sun and major planets is severely hampered by the difficulties inherent in ascertaining the observed coordinates of the centers of such disk-shaped and more or less diffuse objects. Asteroids, on the other hand, can be observed accurately and easily because of their lightpoint images, at least if they are relatively bright. As to their orbits, it is frequently possible to select minor planets that are strongly perturbed by one or several of the major planets, so that optimum conditions exist for the desired mass determinations, provided that well-distributed observations can be obtained. This proviso is important because corrections to the orbital elements of the asteroid in question must be determined simultaneously with the sought-for mass corrections. If the asteroid's heliocentric orbit remains poorly determined, for instance, because it can be observed only near perihelion, then the uncertainties of the orbital elements will unavoidably affect also those of the intimately related mass corrections.

An additional and rather important advantage of the use of asteroids arises from the fact that their own masses are completely negligible, compared to those of the major planets. When dealing with the variations produced in the orbit of an asteroid by the sought-for correction to the mass of a given major planet, one does not have to consider any second-order effects of these orbital changes on the motion of the disturbing or any other major planet, nor does one have to determine or correct the mass of the asteroid. Furthermore, one needs to analyze only the observations of the asteroid, regardless of the number of major planets whose mass corrections are introduced into the observation equations. In contrast to these simplifications, the $O-C$ of any major planet cannot normally be treated separately and independently from those of all the others whose orbits may be affected by the mass corrections involved in the solution. At least when considering substantial mass corrections, one also has to investigate the magnitude of any possibly noticeable second-order effects produced by the first-order orbital variations. It is true that for an asteroid with very large perturbations, a second iteration to the solution may be necessary if the orbital changes produced by the initial correction of the disturbing mass are also substantial, but this additional procedure is still limited to the same set of observations of just one object, even if several planetary masses are being corrected simultaneously.

For the reasons mentioned in the preceding two paragraphs, mass determinations using asteroid observations have long been considered to be more accurate and also more convenient than those using observations of major 
planets and of the Sun. Recently, though, the availability of radar observations of Venus and Mercury has made it possible to increase greatly the accuracy with which the motions and masses of these planets and of Earth can be determined from solutions based on a combination of radar and optical observations of these inner planets and on rigorous numerical integrations of their motions. An even more striking increase in accuracy became apparent when the radio tracking data of the Mariner 2 and 4 space probes were analyzed to determine improved mass values for Venus and Mars, respectively. Obviously the high accuracy with which the very large perturbations produced by the close approaches can be observed by means of Doppler tracking data is superior to the accuracy obtainable from optical observations of even the most favorable asteroid orbits. In passing, it should be noted that the observed orbits of natural satellites can also be used, together with Kepler's third law, to determine the mass of the primary, but that in practice the observational difficulties have tended to limit the attainable accuracy rather severely.

\section{CONSTANTS RELATED TO THE MOTION OF EARTH}

So far only mass variations have been mentioned as affecting the observable motion of an asteroid, aside from any necessary corrections to. its orbital elements. Because all observations are made from the moving Earth, any thorough analysis of the $O-C$ of asteroids approaching relatively close to Earth has to consider also the possible need for correcting some of the elements of Earth's orbit. In this connection, it should be noted again that corrections to the masses of disturbing planets will affect not only the motion of the asteroid under consideration but also the motion of the Earth-Moon barycenter. Consequently, the observation equation coefficients providing for such effects on the $O-C$ may have to be augmented by the relevant (normally much smaller) effects due to the adjusted perturbations of the Earth+Moon orbit. The basic elements for which corrections may be necessary are the mean longitude or mean anomaly at some zero epoch, the longitude of perihelion, the orbital eccentricity, and the obliquity (inclination) of the ecliptic relative to the equator. The mean motion or the semimajor axis, on the other hand, is known much more accurately from long series of observations of the Sun, while the longitude of the node on the equator is intimately connected with the basic definition of the fundamental reference system and thus with the effects of precession. The constants related to the reference frame will be considered in another section. Obviously, the four element corrections to be considered for the orbit of the Earth-Moon barycenter are easily introduced into the observation equations by the same principles as those to the elements of the asteroid orbit. It is evident that especially asteroids of the Eros type will be well suited for actual determinations of such corrections because of the magnification of their effects on the computed positions during all close approaches to Earth. 
The orbital elements just considered are those of the Earth-Moon barycenter, while Earth itself moves about this barycenter in accordance with the Moon's orbital revolutions around Earth. Consequently, the geocentric position of any asteroid is also a function of the so-called constant of the lunar equation, which is the coefficient of the periodic displacement of an object at a distance of $1 \mathrm{AU}$ in the plane of the Moon's orbit, caused by the motion of Earth's center about the barycenter (with the Earth-Moon distance equal to its mean value). This constant $L$ can therefore also be determined from asteroid observations, in particular from $O-C$ of asteroids like Eros or Amor during close approaches. $L$ in tum is related to the Moon/Earth mass ratio $\mu$ through an equation involving also the parallaxes of Sun and Moon. Because these parallaxes were supposed to be known more accurately than $\mu$, many determinations of $\mu$ have been made by deriving $L$ from close-approach residuals of minor planets and then calculating $\mu$ from this equation. Today, however, $\mu$ is one of the primary constants in the newly adopted IAU system of fundamental constants, essentially because of its more accurate and more direct determination from radar observations and space probes, whereas $L$ is now a derived constant. It still enters the computation of geocentric ephemeris positions of planets and asteroids, but it is pointless to try to improve it from observed asteroid residuals in right ascension and declination.

\section{SOLAR PARALLAX AND ASTRONOMICAL UNIT}

A similarly reversed situation exists today with regard to the solar parallax $\pi_{\odot}$ and the astronomical unit, which are related through the definition of $\pi_{\odot}$ as the angle subtended by Earth's equatorial radius $R_{e}$ at a distance of $1 \mathrm{AU}$. (The mean distance of the Earth-Moon barycenter from the Sun does not equal $1 \mathrm{AU}$, but differs from it by a very small and well-defined amount.) Because asteroids are observed from locations on the surface of Earth, and not from its center, the resulting parallactic displacements on the sky are inversely proportional to the geocentric distance, and thus they increase again with the object's approach to Earth. Consequently, asteroids like Eros could be and have been used to determine $\pi_{\odot}$ in this fashion, by the "trigonometric method." The astronomical unit, expressed in meters, could then be calculated from $\pi_{\odot}$ and the known value of $R_{e}$. Today, however, radar observations of Venus are used, for instance, to determine its varying distance from Earth in meters (actually in light-seconds, converted into meters by means of the rather accurately known velocity of light). Because the interplanetary distances are well known in astronomical units, the comparison yields a relatively direct determination of the astronomical unit, and $\pi_{\odot}$ becomes a derived constant.

A very important relation between the mass $m_{\oplus+\varsigma}$ of the Earth-Moon barycenter, the solar parallax $\pi_{\odot}$, and the Moon/Earth mass ratio $\mu$ results from the combination of two equations: the first one governing the acceleration of gravity at the distance $R_{e}$ from Earth's center and the second one representing Kepler's third law for a particle moving around the Sun in 
circular orbit at a distance of $1 \mathrm{AU}$. This relation can be used to compute $\pi_{\odot}$ and thus the astronomical unit from an improved mass $m_{\oplus+\varsigma}$ of Earth and Moon, as obtained from the observed motion of an asteroid such as Eros or Amor. This approach is known as the "dynamical method" for determining $\pi_{\odot}$ and the astronomical unit from asteroid observations because it is actually a determination of $m_{\oplus+\varsigma}$. Because $\mu$ enters the relation between $m_{\oplus+\varsigma}$ and $\pi_{\odot}$ only in the form of a factor $1+\mu$, the uncertainty of the adopted value of $\mu$ was not very significant in these earlier determinations of $\pi_{\odot}$ and the astronomical unit through $m_{\oplus+C}$. In today's IAU system of astronomical constants, however, the astronomical unit as directly determined from radar observations of major planets is a primary constant. Because not only $\pi_{\odot}$ but also $m_{\oplus+\mathbb{C}}$ is a function of the astronomical unit, the $m_{\oplus+\varangle}$ value consistent with the adopted value of the astronomical unit will eventually be used as a derived constant. Presently the IAU system still lists conventional but clearly outdated values for the planetary masses, essentially for practical reasons related to the preparation and publication of ephemerides.

\section{CONSTANTS RELATED TO THE COORDINATE SYSTEM}

Because the comparison of calculated and observed asteroid positions involves the use of a given system of celestial coordinates, so that any changes in the positions of the equator and equinox relative to the stars, as well as in the precessional rates of change, would affect the resulting $O-C$, it is clear that the constants defining orientation and motion of the reference frame can also be determined or corrected by means of asteroids. Moreover, because there may be local distortions and systematic errors even in the best available fundamental star catalogs, which in practice define and represent the adopted reference system in the various areas of the sky, it is possible to determine such errors of a local nature also from the observed positions of asteroids (referred to the catalog stars) as they move across sufficiently large parts of the celestial sphere. If the computed positions are based on excellent and dynamically definitive orbits, their comparison with a sufficient number of observed positions of high accuracy will reveal any local distortions in the right ascensions and declinations of the adopted fundamental system of reference.

Most of the asteroid observations, whether photographic or visual, are relative ones, referred to nearby catalog stars. There is considerable merit, therefore, in making and using absolute meridian circle observations of the first four minor planets, Ceres, Pallas, Vesta, and Juno, for which ephemerides of high internal accuracy are published in the American Ephemeris and Nautical Almanac for each year since 1952. Determinations of the equator, equinox, and the annual precession in longitude from such observations have the advantage of being independent of star catalogs. Compared to similar determinations from observations of the Sun, Mercury, and Venus, the starlike appearance of the asteroids again holds the promise of higher accuracy. Finally, such observations can easily be connected to similar fundamental observations 
of neighboring stars, so that catalogs can be improved at the same time. In any such projects aiming for perfect rigor, corrections to the orbital elements of the asteroids and of the Earth-Moon barycenter will have to be determined with the desired corrections to the constants defining the reference system and its precessional motion.

\section{SOME HISTORICAL NOTES, REMARKS ON FUTURE PROSPECTS}

References to the older determinations of masses and other fundamental constants can be found in a paper by Harkness (1891). Here it may suffice to mention the determination (probably the first) of a planetary mass by means of an asteroid, namely that by Gauss of Jupiter from the motion of Pallas, leading to a result of $1 / 1042.86$ for Jupiter's mass in units of the solar mass. Many astronomical constant determinations made subsequent to Harkness' compilation are listed and discussed in an encyclopedia article by Bauschinger (1920), whereas a number of more recent results, up to the year 1963, are considered by Böhme and Fricke (1965).

Soon after the discovery of 433 Eros in 1898 it became clear that this minor planet was exceptionally well suited for the determination of the solar parallax $\pi_{\odot}$ by the trigonometric method, as well as for the derivation of $m_{\oplus+C}$, because of its rather close approaches to Earth and its observability in all parts of its heliocentric orbit. It was also pointed out by Russell (1900) that because of its substantial perturbations by Mars, this asteroid should be able to yield an accurate determination of the mass of Mars. Because for quite some time Eros has actually been used as the principal tool for determinations of the solar parallax and of $m_{\oplus+C}$, the history of these results may be outlined here in some detail. As to the direct, trigonometric determinations of $\pi_{\odot}$ from Eros, Hinks found $\pi_{\odot}=8 " .807 \pm 0$ ".003 (probable error) from the photographic right ascensions of the 1900-01 close approach (Hinks, 1909) and 8".806 \pm 0 ".004 from the micrometric ones (Hinks, 1910), whereas Spencer Jones (1941) obtained the total result $8.790 \pm 0$ ".001 from the well-prepared 1930-31 approach. The first dynamical determinations of $\pi_{\odot}$, through $m_{\oplus+\mathrm{C}}$, were based on relatively short orbital arcs, but from observations from 1893 (prediscovery positions) through 1914 Noteboom (1921) derived $1 / m_{\oplus+\mathbb{1}}=328370 \pm 68$. This value for the reciprocal of the mass of Earth and Moon was changed only slightly when Witt (1933) found $328390 \pm 69$ from the much longer time interval 1893-1931. The related value of the solar parallax is 8 ".7988 \pm 0 ".0006. The subsequent determination by Rabe (1950) from the more recent time interval $1926-45$, with the results $1 / m_{\oplus+\varsigma}=328452 \pm 43$ and $\pi_{\odot}=8.7984 \pm 0.0004$, essentially seemed to confirm the preceding dynamical results, and thus to maintain the inexplicably large discrepancy with the formally also very accurate trigonometric determination by Spencer Jones. This disagreement became even more puzzling when the first reliable radar measurements of the Earth.Venus distance were all found to point to a solar parallax of very nearly 8.7940 , about halfway between the presumably best 
results from the other two methods. Because of this situation, Rabe and Francis in 1966 undertook a reinvestigation of the Eros motion by means of IBM 7094 integrations using the value $1 / m_{\oplus+C}=328912$, which is consistent with the astronomical unit adopted by the IAU in 1964, extending the comparison with observations through the period 1926-65. It became evident then that the true value of $m_{\oplus+\mathbb{C}}$ was indeed close to $1 / 328912$ (Rabe and Francis, 1967a), and it was found that a conceptual error had led to erroneous mass coefficients in the original 192645 observation equations. The erroneous nature of these coefficients was discovered independently also by Schubart and Zech (1967) when they tried to reconcile the dynamical value of the astronomical unit with the radar measures, after Schubart had first found that the observations of 1221 Amor seemed to call for an Earth+Moon mass fairly consistent with the radar results for the astronomical unit. Rabe's corrected $1926-45$ determination (Rabe, 1967) produced for $1 / m_{\oplus+\varsigma}$ the result $328863 \pm 29$ (mean errors are quoted from here on) from a 13-unknowns solution, whereas from the extended 1926-65 arc Rabe and Francis (1967b) obtained the comparable result $328890 \pm 16$. The gain in accuracy corresponds roughly to the longer arc involved. The most disappointing finding was that the masses of Mars, Venus, and Mercury are very poorly determined from the Eros solutions, contrary to earlier predictions and expectations. This fact has been confirmed even by the as yet most comprehensive and rigorous study of the Eros motion, namely that by Lieske (1968), for which all the 1893-1966 observations have been reduced to the uniform reference system of the FK4. Lieske's result $1 / m_{\oplus+\varangle}=328915 \pm 4$ almost approaches in its small formal error those from radar determinations and space probes, which now point to a true value near $328900 \pm 1$. While the dynamical and radar determinations of the astronomical unit have thus been reconciled, in close agreement with the IAU value 8".79405 for the solar parallax, a full explanation of the still discordant trigonometric result $8.790 \pm 0$ ".001 has not been given as yet.

It appears that the motion of Eros will remain of some value for determinations of $m_{\oplus+\complement}$ even in the future, especially if it should be possible to secure precise radar observations of its distance during close approaches. As to Amor, Schubart (1969) has noted the fact that the lack of observations outside of the perihelion approaches significantly reduces the accuracy of the results for $m_{\oplus+C}$. The motion of 1566 Icarus, on the other hand, from which Lieske and Null (1969) obtained a good determination of the mass of Mercury, will probably remain important for this purpose, at least until space probes make close approaches to this planet. In general, though, it looks as if the future use of asteroids for determinations of the masses of the inner planets will have a strong competition from space probes, as well as from comprehensive adjustments of the (rigorously integrated) orbits and masses of these planets on the basis of combined radar and optical observations of their own motions. As to the latter approach, the relevant investigation by Ash, Shapiro, and Smith (1967), based on a still relatively short time interval, already reveals 
the high accuracy obtainable in this way not only for the planetary masses involved, but even for the Moon/Earth mass ratio $\mu$. As to space probes, the even greater accuracy with which they seem to be able to determine planetary masses is apparent in the result from Mariner 2 for Venus, as quoted by Clemence (1966), and in the one from Mariner 4 for Mars, as obtained by Null (1967).

For most of the asteroids, though, Jupiter is the principal disturbing planet, and many investigators have recently taken up the proposal made in 1873 by Hill (1907) to improve Jupiter's mass on the basis of the particularly large perturbations experienced by certain minor planets in consequence of their closeness to the $2 / 1$ commensurability with respect to Jupiter's mean motion. A recent determination by Klepczynski (1969), for instance, combines the four separate mass corrections obtained from the motions of 10 Hygiea, 24 Themis, 31 Euphrosyne, and 52 Europa into the result $1 / m_{2}=1047.360 \pm 0.004$, with a mean error much smaller than the one appearing in Bec's (1969) determination from Jupiter's ninth satellite: $1 / m_{\chi}=1047.386 \pm 0.041$. On the other hand, an even more recent determination from the disturbed motion of the Hilda group planet 334 Chicago (which approaches Jupiter to within $1.1 \mathrm{AU}$ ) by Scholl (1971) gave the result $1 / m_{x}=1047.325 \pm 0.010$. The minor planet results by Klepczynski and Scholl differ by 3.5 times the larger mean error, and there are other relevant determinations from individual asteroids with formal errors much smaller than the actual differences between some of the results. Nevertheless, it appears that the combination of numerous results from asteroids should eventually give us a Jupiter mass more precise than one can get from satellites.

Because space missions may soon be used also for more accurate determinations of the mass of Jupiter, the major planet whose mass can now be most usefully determined by means of minor planet observations is Saturn. Such a determination, using 944 Hidalgo, has been made by Marsden (1970), who suggests that it would be worthwhile to verify his result $(1 / 3498.5)$ by means of several minor planets with aphelion distances greater than $4 \mathrm{AU}$.

Corrections to the orbital elements of the Earth-Moon barycenter and to the constants defining the equatorial reference system have been included in some comprehensive solutions, especially in those using Eros, sometimes simply to prevent other unknowns from unduly absorbing some of their effects. In this connection, Lieske (1970) found that corrections to the adopted precession in longitude and to Newcomb's rate of change of the obliquity of the ecliptic are not well determined from the Eros data. As to systematic programs using asteroids, the papers by Brouwer (1935), Clemence (1948), Schmeidler (1958), and Petri (1958) may be consulted. First results from meridian observations of the first four numbered planets have been obtained and discussed by Jackson (1968). He finds that the results are considerably less accurate than predicted by Clemence (1948). This is because of the clustering of the observations around opposition and also the less-than-anticipated precision of the individual measures. It is still concluded, however, that meaningful corrections to the 
coordinate system can indeed be obtained from the observations of all four planets during a planned program, if an effort is made to secure observations as close to quadrature as possible. This last requirement is necessary for a satisfactory separation of the corrections to the minor planet orbit from those to the orbit of the Earth. For photographic asteroid observations, the program initiated by Brouwer (1935) has, to a limited extent, been completed by Pierce (1971). Aside from orbital corrections for the 15 minor planets involved, Pierce determines local corrections, $\Delta \alpha$ and $\Delta \delta$, for 54 small areas of the Yale Catalogue zones, and for 60 such areas in the Boss General Catalogue. These catalog corrections are the principal objective of this paper; only rough estimates of the equinox and equator corrections are given, as arithmetic means of all the individual area corrections, and no attempt is made to obtain corrections to Earth's orbit based on volume XIV of the Astronomical Papers of the American Ephemeris. Considering the relatively large and somewhat erratic area corrections obtained, it appears that supplementary results from absolute determinations of equinox and equator, using meridian observations of Ceres, Pallas, Juno, and Vesta, would be of great value in future attempts to disentangle the basic coordinate corrections from the local distortions of star catalogs. In conclusion, it seems fair to say that the further use of asteroids will be of considerable value for future improvements of the fundamental reference frame and for future determinations of the elements of any improved Earth orbit or theory.

\section{REFERENCES}

Ash, M. E., Shapiro, I. I., and Smith, W. B. 1967, Astronomical Constants and Planetary Ephemerides Deduced From Radar and Optical Observations. Astron. J. 72, 338-350.

Bauschinger, J. 1920, Bestimmung und Zusammenhang der Astronomischen Konstanten. Encyklopädie der Mathematischen Wissenschaften, vol. VI, pt. 2, pp. 844-895.

Bec, A. 1969, Détermination de la Masse de Jupiter par l'Étude du Mouvement de son Neuvième Satellite. Astron. Astrophys. 2, 381-387.

Böhme, S., and Fricke, W. 1965, Astronomical Constants. A Survey of Determined Values. Proc. IAU Symp. no. 21, The System of Ȧstronomical Constants, pp. 269-293.

Brouwer, D. 1935, On the Determination of Systematic Corrections to Star Positions From Observations of Minor Planets. Astron. J. 44, 57-63.

Clemence, G. M. 1948, The Value of Minor Planets in Meridian Astronomy. Astron. J. 54, $10-11$.

Clemence, G. M. 1966, Masses of the Principal Planets. IAU Trans. XIIB, 609-614.

Harkness, W. 1891, The Solar Parallax and Its Related Constants, Including the Figure and Density of the Earth. Washington Observations for 1885, app. III. Washington.

Hill, G. W. 1907, On the Derivation of the Mass of Jupiter From the Motion of Certain Asteroids. Collected Mathematical Works, vol. 1, pp. 105-108. (Johnson Reprint Corp., New York, 1965.)

Hinks, A. R. 1909, Solar Parallax Papers no. 7: The General Solution From the Photographic Right Ascensions of Eros, at the Opposition of 1900. Mon. Notic. Roy. Astron. Soc. 69, 544-567.

Hinks, A. R. 1910, Solar Parallax Papers no. 9: The General Solution From the Micrometric Right Ascensions of Eros, at the Opposition of 1900. Mon. Notic. Roy. Astron. Soc. 70, 588-603. 
Jackson, E. S. 1968, Determination of the Equinox and Equator From Meridian Observation of the Minor Planets. Astronomical Papers of the American Ephemeris, vol. XX, pt. 1, pp. 1-131.

Klepczynski, W. J. 1969, The Mass of Jupiter and the Motion of Four Minor Planets. Astron. J. 74, 774-775.

Lieske, J. H. 1968, Mass of the Earth-Moon System From Observations of Eros, 1893-1966. Astron. J. 73, 628-643.

Lieske, J. 1970, On the Secular Change of the Obliquity of the Ecliptic. Astron. Astrophys. 5, 90-101.

Lieske, J. H., and Null, G. W. 1969, Icarus and the Determination of Astronomical Constants. Astron. J. 74, 297-307.

Marsden, B. G. 1970, On the Relationship Between Comets and Minor Planets. Astron. J. 75, 206-217.

Noteboom, E. 1921, Beiträge zur Theorie der Bewegung des Planeten 433 Eros. Astron. Nachr. 214, 153-170.

Null, G. W. 1967, A Solution for the Sun-Mars Mass Ratio Using Mariner IV Doppler Tracking Data. Astron. J. 72, 1292-1298.

Petri, W. 1958, Praktische Auswertung von Meridianbeobachtungen kleiner Planeten. Astron. Nachr. 284, 219-226.

Pierce, D. A. 1971, Star Catalog Corrections Determined From Photographic Observations of Selected Minor Planets. Astron. J. 76, 177-181.

Rabe, E. 1950, Derivation of Fundamental Astronomical Constants From the Observations of Eros During 1926-1945. Astron. J. 55, 112-126.

Rabe, E. 1967, Corrected Derivation of Astronomical Constants From the Observations of Eros 1926-1945. Astron. J. 72, 852-855.

Rabe, E., and Francis, M. P. $1967 a$, Motion of Eros and the Astronomical Unit. Astron. J. 72, 316-317.

Rabe, E., and Francis, M. P. 1967b, The Earth+Moon Mass and Other Astronomical Constants From the Eros Motion 1926-1965. Astron. J. 72, 856-864.

Russell, H. N. 1900, The General Perturbations of the Major Axis of Eros by the Action of Mars. Astron. J. 21, 25-28.

Schmeidler, F. 1958, Über die Bestimmung absoluter Koordinaten-systeme mit Hilfe von Planetenbeobach tungen. Astron. Nachr. 284, 205-218.

Scholl, H. 1971, Correction to the Mass of Jupiter Derived From the Motion of 153 Hilda, 279 Thule, and 334 Chicago. Proc. IAU Colloq. no. 9, The I.A.U.-System of Astronomical Constants (Heidelberg, 1970). Celest. Mech., in press.

Schubart, J., and Zech, G. 1967, Determination of the Astronomical Unit by the Dynamical Method. Nature 214, 900-901.

Schubart, J. 1969, The Minor Planet 1221 Amor. Astron. Astrophys. 2, 173-181.

Spencer Jones, H. 1941, The Solar Parallax and the Mass of the Moon From Observations of Eros at the Opposition of 1931. Mem. Roy. Astron. Soc. 66, pt. 2, 1-66.

Witt, G. 1933, Baryzentrische Ephemeride des Planeten 433 Eros für die Perihelopposition 1930-1931. A stron. Abhand. Ergänzungsh. Astron. Nachr. 9(1), 1-30.

\section{DISCUSSION}

MARSDEN (in reply to a question by Kiang about 1362 Griqua): Because of its libration about the 2:1 resonance, 1362 Griqua does not seem to be as suitable an object for determining the mass of Jupiter as it was thought to be. We attempted to make a determination from the observations of 1935 to 1965 but were unable to obtain a significant correction to the adopted value. The Hill planets, which do not librate and which have longer observational histories, seem to be rather more suitable. 
ALFVEN: Have effects of nongravitational forces been detected in the motion of minor planets?

MARSDEN: No. There are a few cases where it seemed that small systematic trends remained in the residuals after accurate orbit solutions had been made, but I am confident that these are due to errors in the adopted masses of the perturbing planets. I had initially suspected that the motion of 887 Alinda and 944 Hidalgo were affected by nongravitational forces and that these objects were dying cometary nuclei, but it is clear that the residuals may be removed if one makes reasonable changes in the masses of Earth and Saturn. I hasten to add that this is not true in the case of comets, where the residuals are usually very much larger. 